\title{
QUENCH PERFORMANCE AND FIELD QUALITY OF DX DIPOLES FOR RHIC*,
}

\author{
A. Jain", J. Muratore, M. Anerella, G. Ganetis, A. Marone, G. Morgan, A. Prodell, \\ J. Schmalzle, R. Thomas, P. Wanderer, BNL, Upton, NY 11973-5000
}

\section{Abstract}

DX dipoles, with an aperture of $18 \mathrm{~cm}$, are located on each side of the six intersection regions of RHIC. All DX dipoles were tested at $4.35 \mathrm{~K}$ for quench performance, and trained successfully to at least a $10 \%$ margin above the operating field $(4.3 \mathrm{~T}$ at $6.6 \mathrm{kA})$. Field quality in these dipoles was measured at room temperature and at $4.35 \mathrm{~K}$ using a rotating coil system. The measured harmonics were corrected for offsets of the measuring coil from the magnetic axis, determined by a variety of techniques. All harmonics were found to be within acceptable limits.

\section{INTRODUCTION}

The Relativistic Heavy Ion Collider (RHIC) at BNL has six intersection regions. Immediately on each side of these regions is a dipole, named the DX dipole, through which both beams pass. These magnets require a large aperture of $18 \mathrm{~cm}$, operate at $4.3 \mathrm{~T}$, and the magnetic length is $3.75 \mathrm{~m}$. There are 12 of these magnets installed in the accelerator and one more production magnet was built as a spare. The magnet coils have 71 turns in six blocks. A detailed description of magnet features and construction experience can be found in reference [1]. This paper summarizes results of quench and field quality tests.

\section{QUENCH TESTS}

All 13 production DX dipoles were trained to $7.26 \mathrm{kA}$, $10 \%$ above the $6.6 \mathrm{kA}$ operating current. The magnets were tested in liquid helium at $4.35 \mathrm{~K}$ (nom.) and ramped at $100 \mathrm{~A} / \mathrm{sec}$. A summary of the training data for the 12 magnets installed in RHIC is shown in Fig. 1. The filled diamond symbols indicate quench currents, while the open diamonds indicate a ramp to $7.5 \mathrm{kA}$ (the limit of the leads in RHIC) without quenching. Two magnets were not ramped to $7.5 \mathrm{kA}$ due to limitations on test time. The lower final quench for magnet 102 followed an instrumentation-induced quench at high field after reaching $7.5 \mathrm{kA}$.

Constraints on test time permitted thermal cycles only of the spare magnet, number 114. During its initial cooldown, the magnet trained to the operating current in four quenches and to $7.5 \mathrm{kA}$ in twelve quenches. After one cycle to room temperature, four quenches were needed to reach operating current; after a second cycle, three quenches were needed.

Quench location information was available only from the three standard voltage taps. The data indicate that the

\footnotetext{
* Work supported by the US Department of Energy under contract no. DE-AC02-98CH10886.

\# jain@bnl.gov
}

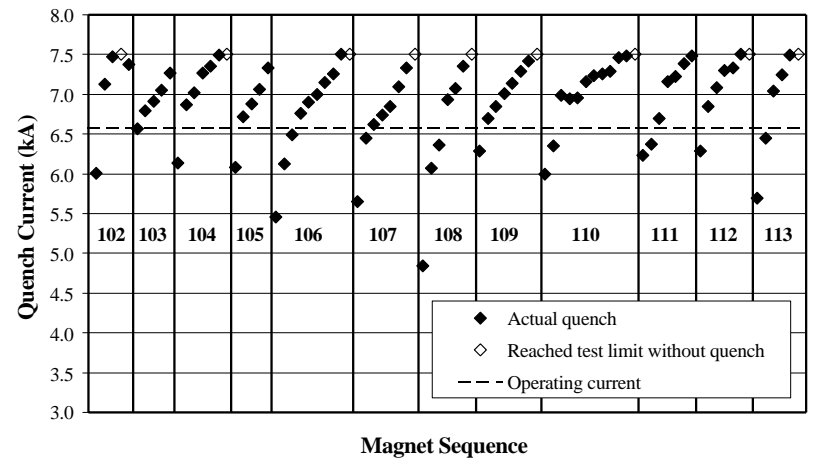

Fig. 1: Quench history of DX dipoles installed in RHIC quenches originated at different locations in both coil halves. Spikes in these signals also indicated significant superconductor motion during most quenches.

\section{FIELD QUALITY MEASUREMENTS}

The field quality is characterized by a set of harmonics, $b_{n}$ and $a_{n}$, defined (in "units") by the relation

$$
B_{y}+i B_{x}=10^{-4} \times B_{0} \sum_{0}^{\infty}\left(b_{n}+i a_{n}\right)\left(\frac{x+i y}{R_{r e f}}\right)^{n}
$$

where $B_{0}$ is the strength of the dipole field and $R_{r e f}$ is a suitable reference radius, chosen to be $60 \mathrm{~mm}$ for the DX magnets. The field quality in all the DX magnets was measured using a rotating coil system comprising of five tangential coils [2] at a radius of $74.1 \mathrm{~mm}$ for warm measurements and $68.5 \mathrm{~mm}$ for measurements at $4.35 \mathrm{~K}$. The length of coils used for both warm and cold measurements was $0.921 \mathrm{~m}$. Harmonics were measured at five axial positions, spaced by one coil length, to obtain the integral harmonics.

\subsection{Warm measurements}

The warm measurements were carried out at three different currents $(10 \mathrm{~A}, 20 \mathrm{~A}$ and $30 \mathrm{~A})$ with the magnet placed horizontally on a level granite table. The "true" harmonics were determined from the slope of a linear fit between the measured normal or skew terms and current, thus subtracting out any remnant fields. The measuring coil was equipped with a gravity sensor, which enabled determination of the dipole field angle with respect to gravity. Several fiducials on the magnet cold mass were optically surveyed during the same set up to correlate the field direction with the fiducials. This information was used to install the magnets into the cryostats in the ring.

\subsubsection{Centering of Data}

Any radial offset of the measuring coil axis with respect to the magnetic axis is a source of error in the 
determination of harmonics. For all the other types of dipoles in RHIC, the Quadrupole Configured Dipole (QCD) method [3] was used for data centering. This method requires a separate measurement with the two magnet coil halves powered with opposite currents. This could not be done for the warm measurements in the DX magnets due to presence of a separate quench protection diode across each coil half.

A variant of the QCD method was devised for centering of data in the warm DX magnets. Measurements were made in the regular dipole mode, and in a "half dipole mode" where only one coil of the magnet was powered. The usual QCD mode data was then synthesized by subtracting the "half-dipole" data weighted by a factor of 2 from the dipole mode data. This technique was tested on the $10 \mathrm{~cm}$ aperture RHIC D0 dipoles and good agreement with the regular QCD method was found.

\subsubsection{Harmonics from warm measurements}

The harmonics measured at the three central positions were used to obtain the average harmonics in the straight section of the magnet. The normal and skew terms from all the five positions were added to obtain the integral harmonics. The results are summarized in Figs. 2(a) and 2(b). The straight section values for individual magnets are shown by crosses and the integral values by open circles. The average values of straight section and integral harmonics are shown by the solid and the dashed lines respectively.

It is seen from Fig. 2 that on an average, the terms unallowed by the dipole symmetry, viz. the odd numbered normal terms $\left(b_{1}, b_{3}\right.$, etc.) and all the skew terms, are quite small in the straight section of the magnet. The only exception is the skew quadrupole, which arises primarily from a size mismatch between the two magnet coils.

A comparison between the straight section and the integral harmonics indicates the contribution from the ends. This is generally seen to be negligible, except for the allowed normal terms and the skew sextupole $\left(a_{2}\right)$. Most of these contributions were found to be from the lead end of the magnet.

\subsubsection{The integral transfer function}

Due to small errors in the axial positioning of the measuring coil, the integral transfer function obtained by

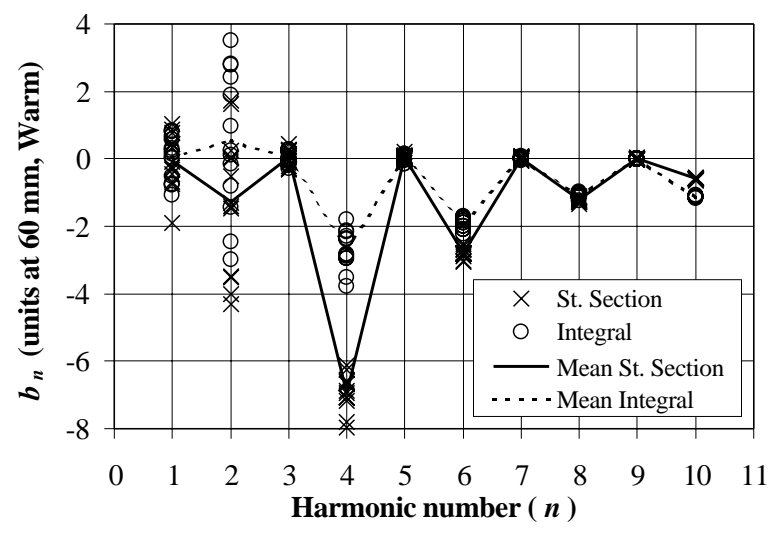

Fig. 2(a): Normal harmonics in DX magnets (warm)

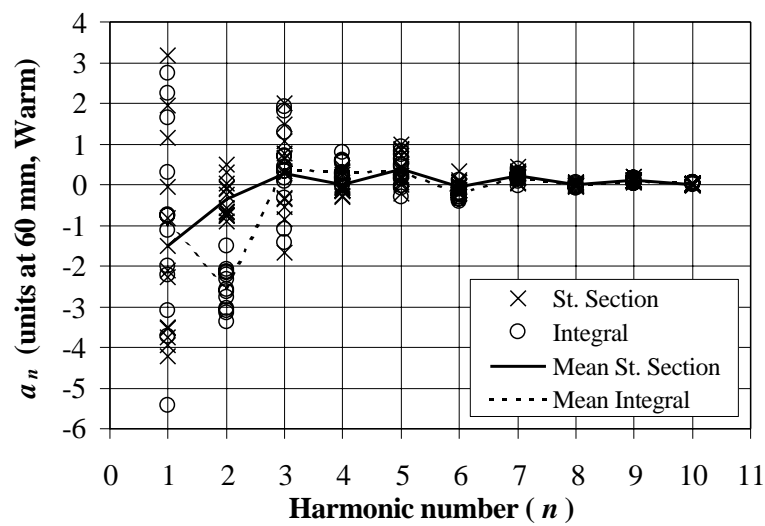

Fig. 2(b): Skew harmonics in DX magnets (warm)

summing the five measurements has a typical accuracy of only $\sim 0.1 \%$. A very long $(10 \mathrm{~m})$ non-rotating coil of $24.3 \mathrm{~mm}$ radius was therefore used for this purpose. The magnet was ramped from $0 \mathrm{~A}$ to $30 \mathrm{~A}$ and the signals from two orthogonal dipole windings were analyzed to obtain the integral transfer function. Fig. 3 gives a trend plot. The mean value is $2.5053 \mathrm{~T} . \mathrm{m} / \mathrm{kA}$ with a standard deviation of $0.03 \%$. The reproducibility of measurements in a given magnet was better than $0.01 \%$.

\subsection{Measurements at $4.35 \mathrm{~K}$}

Field quality was measured at liquid helium temperature, following quench tests in a vertical dewar. The measuring coil built for these measurements was not available during tests of several of these magnets. In such cases, measurements were made with a smaller radius $(34.1 \mathrm{~mm})$ rotating coil. The higher order harmonics were not reliably measured in this case.

At each of the five axial locations of the probe, the harmonics were measured at several currents between $50 \mathrm{~A}$ and $7000 \mathrm{~A}$ during both the upward and the downward sweep of the current. The current was ramped from one value to the next, and then held fixed during the measurements (DC loop). Space limitations above the vertical test facility precluded use of the long coil for cold integral transfer function measurements.

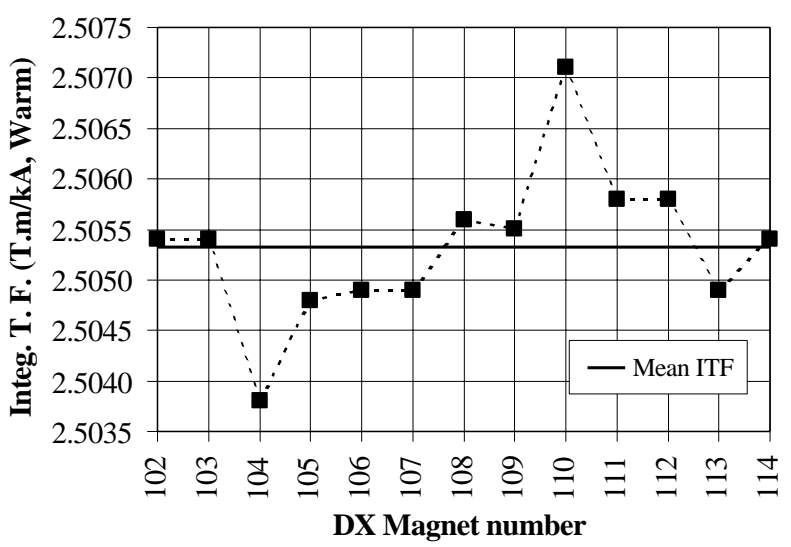

Fig. 3: Integral transfer function (warm) in DX dipoles. 


\subsubsection{Centering of Data}

The regular QCD measurements were used to determine the offsets of the measuring coil. Since there is practically no voltage across the magnet in the superconducting state, the presence of separate diodes across the magnet coils did not prevent the use of this method. These measurements were carried out at $125 \mathrm{~A}$ and $250 \mathrm{~A}$ using two trim power supplies, either immediately before or after each DC loop. The offsets were then used to "center" the measured harmonics.

In some magnets, a pronounced current dependence was seen in the skew terms $a_{1}, a_{3}, a_{5}$, etc. above $\sim 6500 \mathrm{~A}$. As an example, Fig. 4 compares the current dependence of $a_{7}$ and $a_{9}$ in magnets DRX110 (filled symbols) and DRX112 (open symbols). DRX112 shows little current dependence in these harmonics, as expected, but this is not the case with DRX110. Magnet to magnet variation in the behavior of such higher order skew terms is very unusual. A closer look at the magnitudes of changes in various harmonics suggested that these could be explained by a

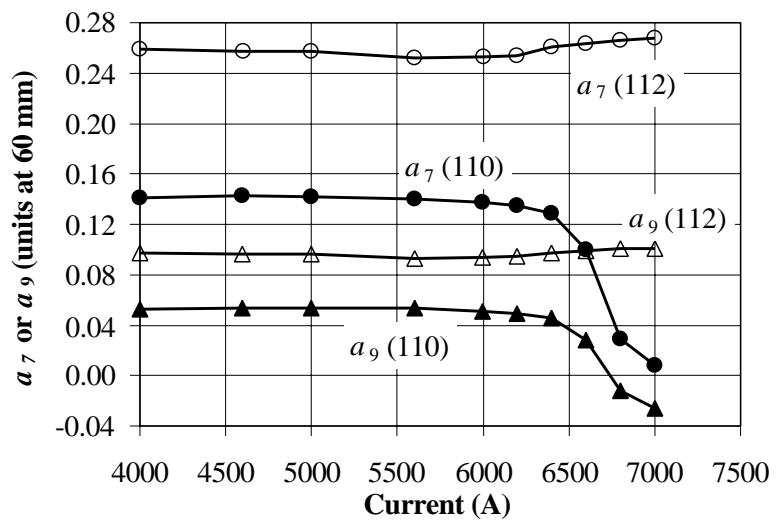

Fig. 4: Current dependence of $a_{7}$ and $a_{9}$ in a straight section region. The dependence in DRX110 at high fields is believed to be an artifact due to a small transverse motion of the magnet.

small $(\sim 0.5 \mathrm{~mm})$ motion of the magnet at high fields relative to the measuring coil. In order to account for such a motion in the analysis, harmonics were first obtained at low fields using centers from the QCD measurements. A second pass was then made with centers determined such that the current dependence in very high order harmonics $\left(a_{9}\right.$ and $a_{11}$ ) was eliminated. This procedure eliminated current dependence in other lower order skew terms as well, except the skew quadrupole, which could have a real current dependence due to local top-bottom asymmetries in the iron yoke weights.

A typical excitation curve is shown in Fig. 5. The standard deviation of the straight section transfer functions measured in 6 magnets is $\sim 0.03 \%$. The normal and skew harmonics at $6600 \mathrm{~A}(4.33 \mathrm{~T})$ in these six magnets are shown in Figs. 6(a) and 6(b). Changes in harmonics due to quench and thermal cycle were studied in one magnet and were generally $\sim 0.1$ unit or smaller.

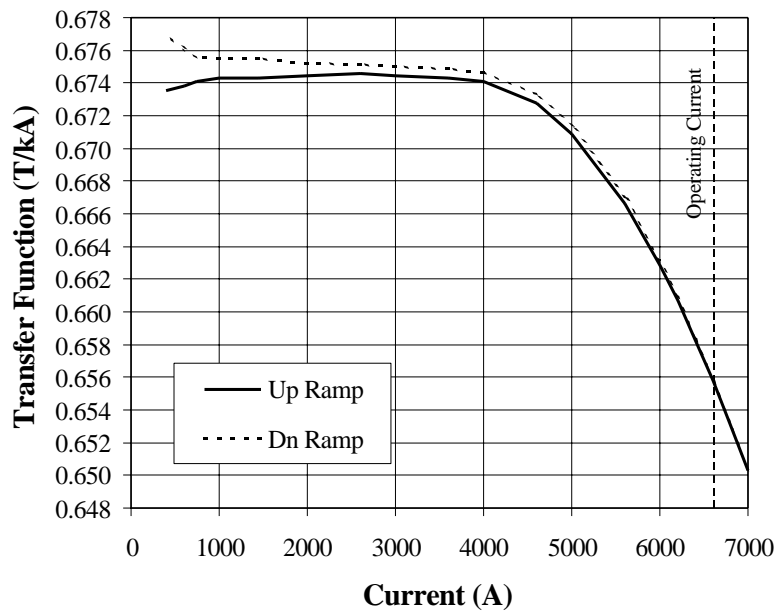

Fig. 5: Excitation curves in a typical magnet, DRX113.

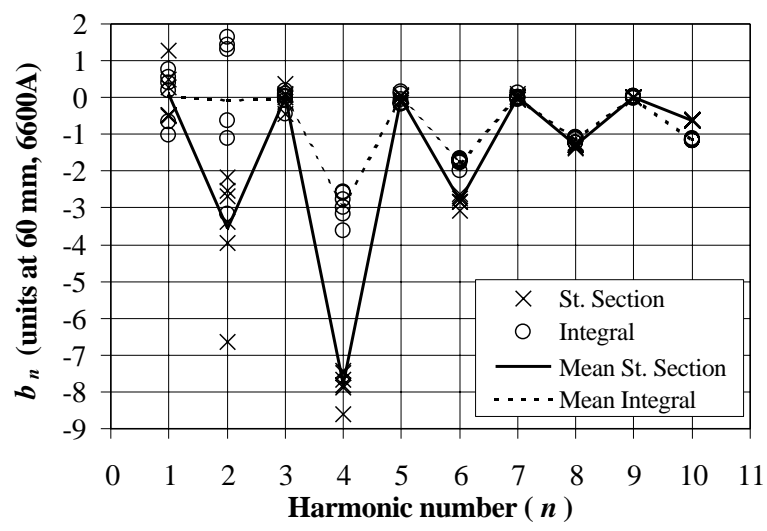

Fig. 6(a): Normal harmonics in DX magnets (6600A)

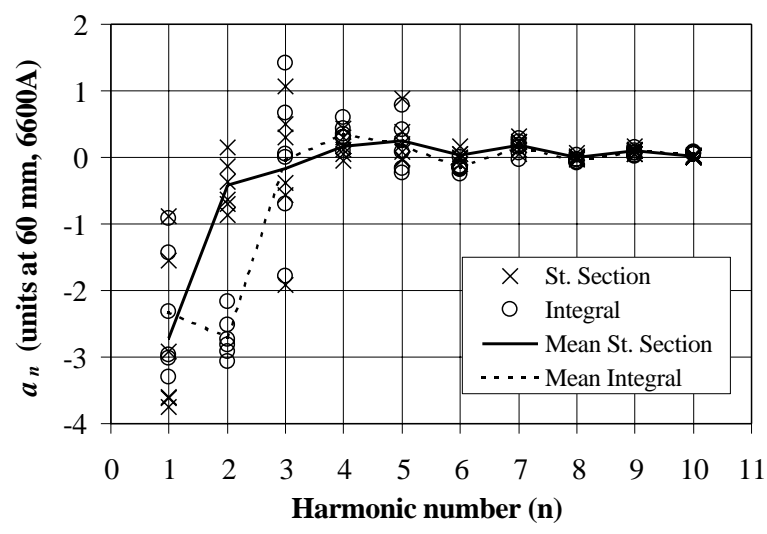

Fig. 6(b): Skew harmonics in DX magnets (6600A)

\section{REFERENCES}

[1] J. Schmalzle, "DX Magnet R\&D, Prototype Construction and Magnet Production”, BNL Note RHIC-MD-279, October 27, 1998.

[2] J. Herrera et al., "Measurement of the Magnetic Field Coefficients of Particle Accelerator Magnets", Proc. of 1989 Particle Accel. Conf., IEEE Catalog No. 89CH2669-0, pp. 1774-6.

[3] C.R. Gibson et al., "Locating the Magnetic Center of the SSC CDM Using a Temporary Quadrupole Field", Proc. 1992 Applied Superconductivity Conf., Chicago, Aug.23-28, 1992 in IEEE Trans. on Applied Superconductivity, 3, No.1, p.646-9 (March, 1993). 